University of Nebraska - Lincoln

DigitalCommons@University of Nebraska - Lincoln

May 1995

\title{
THE PARAMAGNETIC CORRELATION LENGTH OF Mn THIN FILMS
}

D.N. Mcllroy

University of Nebraska-Lincoln

Peter A. Dowben

University of Nebraska-Lincoln, pdowben@unl.edu

Follow this and additional works at: https://digitalcommons.unl.edu/physicsdowben

Part of the Physics Commons

Mcllroy, D.N. and Dowben, Peter A., "THE PARAMAGNETIC CORRELATION LENGTH OF Mn THIN FILMS" (1995). Peter Dowben Publications. 159.

https://digitalcommons.unl.edu/physicsdowben/159

This Article is brought to you for free and open access by the Research Papers in Physics and Astronomy at DigitalCommons@University of Nebraska - Lincoln. It has been accepted for inclusion in Peter Dowben Publications by an authorized administrator of DigitalCommons@University of Nebraska - Lincoln. 


\title{
THE PARAMAGNETIC CORRELATION LENGTH OF Mn THIN FILMS
}

\author{
D. N. MclLroy and P. A. Dowben
}

Dept. of Physics, University of Nebraska-Lincoln, Lincoln, NE 68588-0111

\section{Abstract}

We have utilized Ginzberg-Landau mean field theory to analyze the branching ratio and soft $X$-ray magnetic circular dichroism measurements of the $L_{3}$ and $L_{2}$ adsorption edges of thin $\mathrm{Mn}$ overlayers on ferromagnetic fcc $\mathrm{Co}(001)$ at room temperature. We have determined a short range magnetic correlation of $4.25 \AA$ and that the long range magnetic magnetic order has a decay length of $1.29 \AA$. These results indicate that the first monolayer of $\mathrm{Mn}$ ferromagnetically couple with the Co substrate. The paramagnetic $\mathrm{Mn}$ layer exhibits short range magnetic order, but lacks long range magnetic order. Due to the exponential decrease of both the branching ratio and dichroism signals, we have concluded that the magnetization of the $\mathrm{Mn}$ overlayer is substrate induced.

Ginzberg-Landau formalism has been applied to the study of thin films with surprising success. ${ }^{1-13}$ In fact, for large moment systems, such as the rare earths, this mean field approach to characterizing the influence of the substrate upon the magnetic ordering of a thin film works well and fails only at submonolayer coverages. Of all the $3 d$ magnetic systems, only $\mathrm{Mn}$ has a large enough local moment to be considered (like the rare earths) a local moment system. Therefore, $\mathrm{Mn}$ thin films should also be amenable to Ginzburg-Landau theory.

While some theories strive to better understand the underlying microscopic Hamiltonian of magnetic systems, a more applied approach which extracts macroscopic magnetic properties is of greater use to the materials science and industrial communities. We have taken the later approach to understand the results of $\mathrm{X}$-ray magnetic circular dichroism (XMCD) experiments of thin paramagnetic $\mathrm{Mn}$ overlayers on $\mathrm{Co}(001)$. Since the experiment was conducted far away from $T_{C}$, a mean field approach using Ginzburg-Landau theory is justified for modeling this system.

We will only briefly touch on the specifics of the experimental since they have been discussed in detail elsewhere.14 Soft X-ray adsorption spectroscopy with circularly polarized light was conducted on ultra thin films ranging from 1 to 14 monolayers (ML) of $\mathrm{Mn}$ on $\mathrm{Co}(001)$ at room temperature. The experiments were conducted at the Synchrotron Radiation Center, Stoughton WI, on the 10M-TGM beamline. Film growth and subsequent analysis was performed in a UHV chamber at a base pressure of $5 \times 10^{-11}$ Torr. The adsorption cross-section at the $L_{3}$ and $L_{2}$ edges of $\mathrm{Mn}$ and $\mathrm{Co}$ were obtained from total electron yield with photon spin parallel $\left(\sigma^{+}\right)$ and anti-parallel $\left(\sigma^{-}\right)$to the net magnetization vector within the sample. The analysis requires that the magnetization be "in plane", which is the case for this experiment. All of the data was acquired in remanence.

In a previous work, ${ }^{2}$ Ginzburg-Landau theory was applied to the branching ratio of the 5p shallow cores of Gd and Tb thin films in order to determine the spatially dependent magnetization $\boldsymbol{M}(\mathrm{r})$ as a function of film thickness. In addition to the rare earths, Gindzburg-Landau has been successfully applied to other films, alloys, and ferromagnetic multilayers. ${ }^{2-13}$ The excellent agreement between experiment and theory has prompted us to apply this theory to the system of $\mathrm{Mn} / \mathrm{Co}(001)$. Since the 
development of this theory has been discussed in great detail elsewhere, 2,3 we will only present a brief review of the formalism leading to the final relationship relating the local magnetic ordering of paramagnetic films to film thickness. We begin with the free energy per unit volume in the Ginzburg-Landau formulation $11,13,15$

$$
f(\mathbf{r})=f_{0}(\mathbf{r})+1 / 2 A[M(\mathbf{r})]^{2}+B / 4[\mathbf{M}(\mathbf{r})]^{4}-1 / 2 \mathbf{M}(\mathbf{r}) \cdot \mathbf{H}^{m}-\mathbf{M}(\mathbf{r}) \cdot \mathbf{H}+1 / 2 \mathrm{C}[\nabla \mathbf{M}(\mathbf{r})]^{2}
$$

The first term $f_{o}(r)$ is the free energy per unit volume in the absence of an applied field, and the magnetization $\mathbf{M}(\mathbf{r})$ is the order parameter. The fields $\mathbf{H}^{\mathrm{m}}$ and $\mathbf{H}$ are the demagnetization and applied field, respectively. The Ginzburg-Landau coefficients $A$, $B$, and $C$ are temperature-dependent parameters.

In the Gaussian approximation, the static magnetization $M_{0}(r)$ is found by solving $\delta F / \delta M=0$. This approximation is valid except for in the immediate vicinity of the critical point. Applying this approximation and setting $\mathbf{H}=0,11,16,17$ we arrive at the differential equation

$$
A M_{0}(y)+B M_{0}(y)^{3}-C\left(d^{2} M_{0}(y) / d y^{2}\right)=H^{m}{ }_{0}(y) \text {, }
$$

where the coordinate $y$ is the distance of the considered plane in the overlayer film to the interface. Hence $0 \leq y \leq z$, where $z$ is the film thickness. From symmetry considerations, we do not expect $M_{0}(r)$ to depend on in-plane coordinates. Therefore, $\mathrm{H}^{\mathrm{m}} \mathrm{o}(\mathrm{y})$ is the component of $\mathbf{H}^{\mathrm{m}}$ along the magnetization direction. For our thin paramagnetic films of $\mathrm{Mn}$ the magnetization is in-plane and therefore the demagnetization field $H^{m}$ is negligible. So setting $\mathbf{H}^{\mathrm{m}}$ to zero in Eq. (2) and integrating, we arrive at the differential equation

$$
1 / \kappa^{2}[d M(y) / d y]^{2}=[M(y)]^{2}+b[M(y)]^{4}+a,
$$

where $b=B / 2 A$ and $a$ is a constant of integration. the correlation length, which is dependent on bulk properties of the overlayer, is $\mathrm{K}^{-1}$ and is related to the short-range magnetic order. ${ }^{16}$ The final integration of Eq. (3) can be cast in the form of an incomplete elliptic integral of the first kind.

At this time we will only present the boundary conditions and the final solution since the complete derivation has been presented elsewhere, ${ }^{1}$

$$
\begin{aligned}
& {\left[(\kappa \gamma)^{-2}-1\right][M(0)]^{2}-b[M(0)]^{4}=a} \\
& {[(\kappa \lambda)-2-1][M(z)]^{2}-b[M(z)]^{4}=a}
\end{aligned}
$$

In the above equations $\lambda$ is a length constant which describes the free surface and is analogous to a free surface extrapolation length, $\gamma$ is similar to $\lambda$, but characterizes the interface, $M(0)$ is the static magnetization at the interface, $M(z)$ is the static magnetization of the free surface, where we have made the substitution $z=y$, and again $a$ is a constant of integration. The final solution for in-plane magnetization of a paramagnetic film is,

$$
M_{\|}(z) \equiv R \exp (-\kappa z)
$$

where $z$ is the film thickness, $R$ is a constant, and $\kappa^{-1}$ is again the magnetic correlation length and is equal to $(C / A)^{1 / 2}$. The above relationship is valid for $T>T_{c}$. Since we 
are well above the Neèl temperature of bulk $M n$ (for $\alpha-M n T_{c}=95 K$ ), and furthermore, finite size scaling arguments will only serve to reduce the Neèl temperature of a thin film, this is a valid approach.

If $\mathrm{Eq}$. (7) accurately describes the magnetization near the free surface, then $\kappa \lambda=1$. If the free surface magnetization is larger than predicted by an exponential decay then $\kappa \lambda>1$, corresponding to a small value of $\left.\right|^{\prime}(z) I_{y=z}$. However, $M(z)<M(y)$ will still hold for $y<z$, thus the free surface is weakly enhanced. For the case where $0<\kappa \lambda<1,\left|\mathrm{M}^{\prime}(z)\right|_{y=z}$ is of a larger size than for a simple exponential decay and corresponds to the suppression of surface magnetization. For the two situation where $-1<\kappa \lambda<0$ and $\kappa \lambda<-1$ there is an increase in $M(z)$ relative to layers beneath the surface, where the first condition corresponds to a live surface and the second condition to a strongly enhanced surface, respectively.

In Fig. 1 we present the branching ratio of the $L_{3}$ and $L_{2}$ adsorption edges of $\mathrm{Mn} / \mathrm{Co}(001)$ as a function of thickness as determined by total electron yield, as well as, the fit of Eq. (7) to the experimental data. The data is represented by symbols while the solid line through the data represents the fit of Eq. (7). From inspection of Fig. (1) we find that there is excellent agreement between the experimentally determined branching ratio and the fitting of $\mathrm{Eq}$. (7).

From the fit of the branching ratio data of $\mathrm{Mn} / \mathrm{Co}(001)$ (solid line in Fig. (1)) we obtained a correlation length of $4.25 \AA$ which indicates that $\mathrm{Mn}$ adatoms couple strongly with their nearest neighbors. Such a large correlation length of several atomic diameters is indicative of an itinerant electron exchange mechanism. This is a highly itinerant system exhibiting local order. The exponential decay of the branching ratio suggests that there is paramagnetic ordering of the interface layer of the Mn film as a consequence of the magnetic field produced by the ferromagnetic substrate. The short range correlation length of palladium on nickel was found to be $5.0 \AA, 4$ which is about the same number of atomic diameters as we observe for paramagnetic $\mathrm{Mn}$. While for the rare-earths possessing much larger moments, paramagnetic correlation lengths of only about one atomic diameter have been observed. ${ }^{1}$ The similarities between $\mathrm{Pd}$ and $\mathrm{Mn}$ extend beyond their similar large values of their short range correlation lengths, $\mathrm{Pd} / \mathrm{Co}$ multilayers are widely recognized magnetically enhanced systems. ${ }^{18-21}$ Similarly, $\mathrm{Mn}$ has been observed to posses a large magnetic moment in alloy compounds containing Co $\left(2.9 \mu_{\mathrm{B}}\right.$ for $\mathrm{Mn}$ in $\left.\mathrm{MnCoGe}\right) .22$

The horizontal line at 2.0 on the $y$-axis of Fig. 1 represents the idealized branching ratio in the absence of paramagnetic order, where the two dashed lines

above and below indicate the range of crystal-field variations. ${ }^{23}$ The large deviation of the measured branching ratio at thick coverages $(\sim 0.57)$ from the ideal value of 2.0 may be a consequence of crystal field effects or residual paramagnetic ordering due to a "live" magnetic surface. Since crystal-field effects should be temperature independent, measurements of the branching ratio at different temperatures would determine if the deviation of the branching ratio from the ideal value of 2.0 at thick coverages is, in fact, a consequence of crystal-field effects, or due to a live surface layer (as indicated by equation 6).

A fit of Eq.(7) to the MCD data of $\mathrm{Mn} / \mathrm{Co}(001)$ is presented in Fig. 2. Again a very good fit is obtained with a correlation length of $1.29 \AA$. This is not the short range paramagnetic correlation length derived above. The MCD results provide information about the long range magnetic ordering. Therefore, the correlation length obtained from the fit of Eq.(7) provides information about the degree of long range order and a 
value of $1.29 \AA$ indicates that the Mn overlayer does not exhibit long range magnetic order.

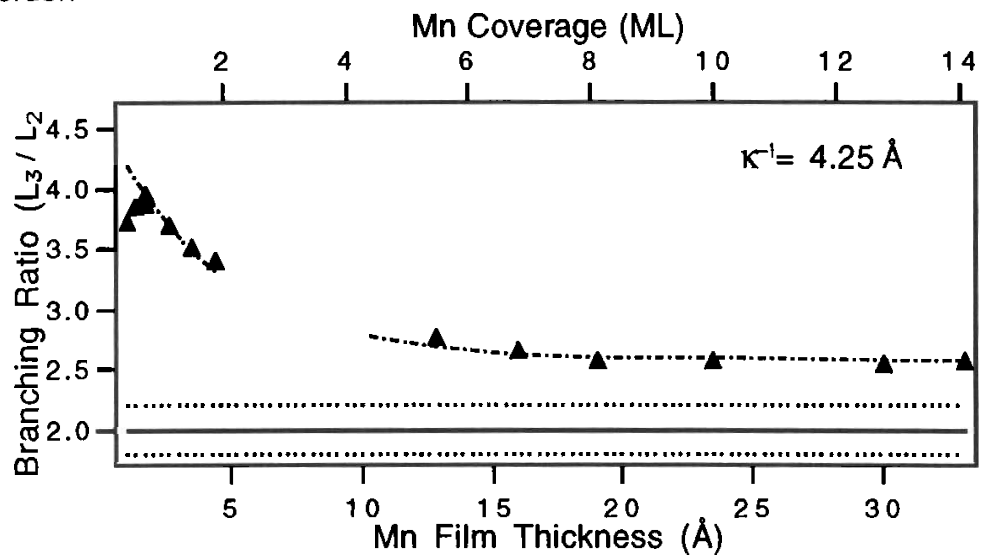

Figure 1. The branching ratio of the $L_{3}$ and $L_{2}$ adsorption edges of $M n / f c c . C o(001)$ as a function of $\mathrm{Mn}$ coverage. The symbols are the experimental data and the dashed line through the data points is the fit of Eq. (7). The horizontal line at 2.0 on the $y$-axis is the idealized branching ratio in the absence of inversion. The dashed lines represent estimates of variances from the value of the idealized branching ratio due to crystal-field effects. A short range magnetic correlation length of $4.25 \AA$ was determined from the fit of $\mathrm{Eq}$. (7).

To reconcile the two different correlation lengths for the branching ratio and MCD measurements we need either to invoke two different definitions for the magnetism (for example static versus dynamic magnetization), or in some way reconcile the two measurements. Shull and Wilkinson have determined the average paramagnetic moment of $\mathrm{Mn}$ to be $\sim 0.5 \mu_{\mathrm{B}}$ from neutron diffraction, 24 while the magnetic susceptibility 25 and multiplet splitting ${ }^{26}$ in photoemission suggests a larger moment of $2.4-2.5 \mu_{\mathrm{B}}$ for paramagnetic $\alpha-\mathrm{Mn}$. Thus the larger localized moments exist on the time scale of $10^{-12}$ to $10^{-15}$ seconds, ${ }^{26}$ while concurrently the net magnetization of the film may be quite small. Some forms of time dependence can be accounted for in mean field theory $y^{13}$ and would apply to the branching ratio data (Fig. 1), but not to the MCD data (Fig. 2) which would be time independent. Therefore, with a difference in the dependence upon the dynamic magnetization, different correlation lengths can be observed.

The cobalt ( as in the case of $\mathrm{Pd} /$ Co multilayers) clearly induces substantial moments in the Mn overlayer due to the presence of the large magnetic field generated by the ferromagnetically ordered Co substrate. The first layer of the $\mathrm{Mn}$ film, in particular, couples with the substrate. At low Mn coverages $(<2 \mathrm{ML})$, the substrate and the $\mathrm{Mn}$ overlayer interact strongly, but as the film becomes thicker ( $\geq 2 \mathrm{ML}$ ) the interaction becomes weaker as the screening of the magnetic substrate increases with increasing $\mathrm{Mn}$ coverage.

Let us suggest that at the interface we have quasi-alloying between two $\mathrm{Mn}$ atoms and a $\mathrm{Co}$ atom i. e., two neighboring $\mathrm{Mn}$ atoms couple to form a pair, which in turn, couples with a single $\mathrm{Co}_{\mathrm{O}}$ atom of the surface. It has been shown that as $\mathrm{Mn}-\mathrm{Mn}$ pairing within $\mathrm{Mn}$ alloy quasi-crystals increases, an enhancement of the local magnetic moments of the $\mathrm{Mn}$ atoms occurs. ${ }^{27-31}$ An enhancement of the local magnetic moment of the $\mathrm{Mn}$ adatoms within the first few monolayers may be 
occurring, which in turn increases the ferromagnetic coupling of the $\mathrm{Mn}$ to the Co substrate. While we are not arguing that this is the case, it does suggest a novel view towards understanding these results of $\mathrm{Mn} / \mathrm{Co}(001)$.

The fact that the branching ratio does not reach the ideal value of 2.0 is either a consequence of residual order due to crystal field effects, 32 or enhanced local surface magnetization (not necessarily ferromagnetism). If this is not due to crystal field effects, then our boundary conditions from Eqs. (5) and (6) would suggest that the magnetization of the free surface of the Mn thin film is weakly enhanced, at least in so far as the local moment measured on the time scale of photoemission. This is consistent with the crystal field splitting results of McFeely and coworkers. 26

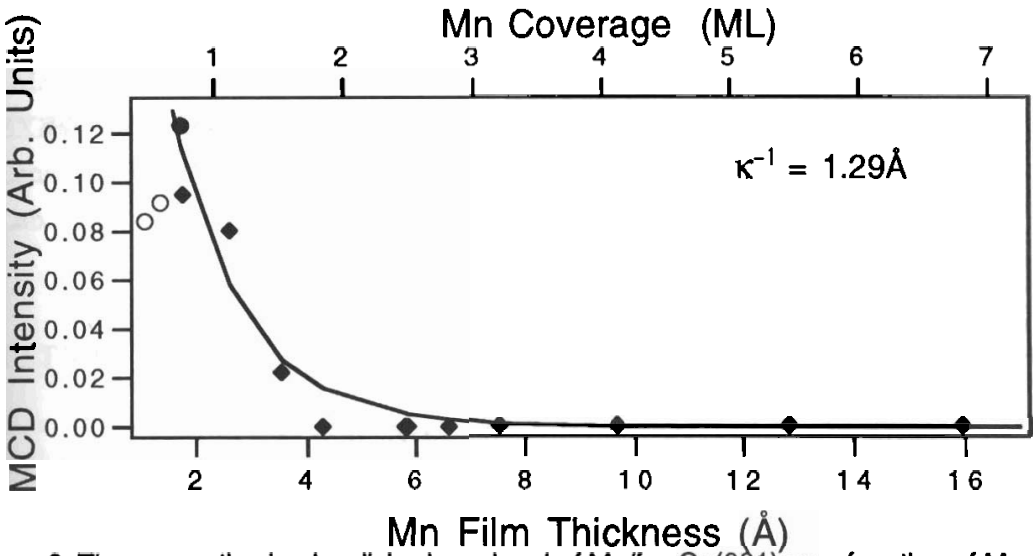

Figure 2. The magnetic circular dichroism signal of $\mathrm{Mn} / \mathrm{fcc} \mathrm{Co}(001)$ as a function of $\mathrm{Mn}$ coverage. The symbols are the experimental data and the solid line through the data points is the fit of Eq. (7). A long range magnetic correlation length of $1.29 \AA$ was determined from the fit of Eq. (7). The open symbols correspond to the increase in the MCD signal upon the initial deposition of Mn.

Upon examination of Fig. 2 we see that the MCD signal drops off very quickly and reaches a value of zero at about $4.25 \AA(\sim 2 \mathrm{ML})$. From these results we can conclude that the long range magnetic order is all but destroyed by the completion of the second monolayer of $\mathrm{Mn}$. This is substantiated by the relatively short long range correlation length of $1.29 \AA$ which we extracted from the fit of the MCD data in Fig. 2. O'Brien and Tonner indicated that while they still observed fcc $(1 \times 1)$ LEED spots at

$2 \mathrm{ML}$ of $\mathrm{Mn}$, they noted that background intensity constantly grew with Mn coverage. 14 This may indicate the presence of disorder within the $\mathrm{Mn}$ film and magnetic correlation on a very short time scale, or magnetic order with no net magnetic moment (antiferromagnetism). This disorder would contribute to the break down of the long range magnetic order, which in turn would strongly attenuate the MCD signal. This view agrees well with the MCD measurements and the rather short long range correlation length of $1.29 \AA$ we obtained from the application of Ginzburg-Landau theory to the MCD data. We therefore conclude that the Mn film may be structurally and magnetically order at coverages up to $2 \mathrm{ML}$, but begins to structurally disorder once this coverage is exceeded, and as a consequence, the long range magnetic order is destroyed. The poor agreement between the theory and the experimental measurements below one monolayer is understandable because these coverages fall below the percolation limit where Ginzburg-Landau theory is expected to fail. 
To summarize, we have interpreted the results of branching ratio and $X$-ray magnetic circular dichroism .measurements of the $L_{3}$ and $L_{2}$ adsorption edges of $\mathrm{Mn} / \mathrm{fcc}$ Co (001) using Ginzburg-Landau theory. Upon examination of the branching ratio measurements we determined a value of $4.25 \AA$ for the short range correlation length. This indicates that the $\mathrm{Mn}$ film is locally ordered and is coupled to the Co substrate. On the other hand, the rather small value of $1.29 \AA$ for the long range correlation length obtained from the MCD data, in conjunction with the strong attenuation of the MCD signal, suggests that the Mn may be structurally and magnetically ordered up to only $2 \mathrm{ML}$, or less, of $\mathrm{Mn}$, but once this thickness is exceeded, the structure of the $\mathrm{Mn}$ film becomes increasingly disordered which destroys the long range magnetic order. In our model, the $\mathrm{Mn}$ exhibits magnetic order which is substrate induced, but is otherwise paramagnetic.

\section{References}

1. P. A. Dowben, D. LaGraffe, Dongqi Li, A. Miller, L. Zhang, L. Dottl, and M. Onellion, Phys. Rev. B 43,3171 (1991).

2. A. Miller and P. A. Dowben, J. Phys.: Condens. Matter 5, 5459 (1993).

3. J. Mathon, J. Phys. F: Met. Phys. 16, L217 (1986).

4. J. Mathon, J. Phys. F: Met. Phys. 16, 669 (1986).

5. J. Mathon and G. Bergmann, J. Phys. F: Met. Phys. 16, 887 (1986).

6. K. Binder and P. C. Hohenberg, Phys. Rev. B 6, 3461 (1972).

7. D. M. Edwards, J. Mathon, and E. P. Wohlfarth, J. Phys. F: Met. Phys. 5, 1619 (1975).

8. D. M. Edwards, J. Mathon, and E. P. Wohlfarth, J. Phys. F: Met. Phys. 3, 161 (1973).

9. S. Coutinho, D. M. Edwards, and J. Mathon, J. Phys. F: Met. Phys. 143, I143 (1983).

10. R. E. Camley, Phys. Rev. B 35, 3608 (1987).

11. F. Fishman, F. Schwabl, and D. Schwenk, Phys. Lett. 121A, 192 (1987).

12. R. E. Camley and D. R. Tilley, Phys. Rev. B 37, 3413 (1988).

13. D. Schwenk, F. Fishman, and F. Schwabl, Phys. Rev. B 38, 11618 (1988).

14. W. L. O'Brien and B. P. Tonner, Phys. Rev. B 5, 2963 (1994).

15. U. Gradmann and R. Bergholz, Phys. Rev. Lett. 52, 771 (1984).

16. C. Domb and M. S. Green. Phase Transitions and Critical Phenomena (Academic. New York, 1976), Vol. 5A.

17. E. M. Liftshitz and L. P. Pitaevski, Statistical Physics, Part 2 (Pergamon,Oxford, 1980), p. 182.

18. S. Blügel, M. Weinert, and P. H. Dederichs, Phys. Rev. Lett. 60, 1077 (1988); S. Blügel, B. Dittler, R. Zeller, and P. H. Dederichs, Appl. Phys. A 49, 547 (1989).

19. R. Wu, C. Li, and A. J. Freeman, J. Magn. and Magn. Mater. 99, 71 (1991).

20. K. Miura, H. Kimura, S. Imanaga, and Y. Hayafuji, J. Appl. Phys. 72, 4826 (1992).

21. S. K. Kim, J. S. Kang, J. I. Jeong, Y. M. Koo, H. J. Shin, and Y. P. Lee, J. Appl. Phys. 72 , 4986 (1992); Y. P. Lee, S. K. Kim, J. S. Kang, J. I. Jeong, J. H. Hong, and H. J. Shin, J. Magn. and Magn. Mater. 126, 316 (1993).

22. S. Niziol, A. Bombik, W. Bazela, A. Szytula, and D. Fruchart, J. Magn. and Magn. Mater. 27,281 (1982).

23. We have used the calculated crystal-field variations Tb/Ni(110) from Ref. 2 in order to obtain an idea of what reasonable variations we might expect for $\mathrm{Mn} / \mathrm{Co}(001)$.

24. C. G. Shull and M. K. Wilkinson, Rev. Mod. Phys. 23, 100 (1953).

25. H. Nagasawa and M.Uchinami, Phys. Lett. 42A, 463 (1973).

26. F. R. McFeely, S. P. Kowalczyk, L. Ley, and D. A. Shirley, Solid State Commun. 15, 1051 (1974).

27. J. J. Hauser, H. S. Chen, and J. V. Waszczak, Phys. Rev. B 33, 3577 (1986).

28. F. L. A. Machado, W. G. Clark, L. J. Azevedo, D. P. Yang, W. A. Hines, J. I. Budnick, and M. X. Quan, Solid State Commun. 61, 145 (1987).

29. K. Edagawa, H. Ino, S. Nasu, K. Kimura, S. Takeuchi, T. Shinjo, K. Koga, T. Shimizu, and H. Yasuoka, J. Phys. Soc. Jpn 56, 2629 (1987).

30. R. Bellisent, F. Hippert, P. Monod, and F. Vigneron, Phys. Rev. B 36, 5540 (1987).

31. M. E. McHenry, D. D. Vvedensky, M. E. Eberhart, and R. C. O'Handley, Phys. Rev. C 37, 10887 (1988)

32. J. B. Goedkoop, PhD Thesis, Katholiek Universitiet te Nijmegen (1989); J. B. Goedkoop, M. Grioni, and J. C. Fuggle, Phys. Rèv. B 43, 1179 (1991). 